\title{
Hyperosmolar Solution Reduces Apoptosis in the Acute Spinal Cord Injury Model in Rats
}

\author{
Samuel Dobrowolski ${ }^{1}$, Gustavo Rassier Isolan ${ }^{2}$ and Guilherme Lepski ${ }^{3 *}$ \\ ${ }^{1}$ Department of Neurosurgery, Faculdade Evangélica do Paraná, Curitiba, Brazil \\ ${ }^{2}$ Department of Neurosurgery, Universidade Federal do Rio Grande do Sul, Porto Alegre, Brazil \\ ${ }^{3}$ Department of Neurosurgery, University Eberhard Karls, Tübingen, Germany
}

\section{Introduction}

Spinal cord injury is a devastating clinical condition that often causes permanent incapacity, especially in the younger population. The clinical relevance of SCI justifies the scientific interest in the pathophysiology of SCI, as well as in protective effects and development of treatment options.

Although the primary lesion caused by traumatic injury to the spinal cord is considered intractable, the secondary lesions and consequent clinical manifestations can be diminished considerably with early administration of steroids or by surgical decompression of the injured segment, removal of bony fragments and stabilization [1-3].

The extent of the neurologic deficits caused by the lesion is determined by two main factors: the primary mechanical insult and the secondary insult caused by inflammation, compression and ischemia [4-6]. The primary lesion is caused by the trauma itself and involves cellular death and tissue necrosis, independently of biological factors. The mechanisms underlying secondary lesions involve activation of inflammation, tissue ischemia, reperfusion deficits, edema, lipidic peroxidation, calcium influx, and particularly apoptosis. The secondary lesions constitute the main target for the development of novel therapeutic approaches [7-9]. Among these novel strategies, new anti-apoptotic agents are being developed, in the hope that their administration might limit tissue damage and increase the potential for clinical recovery [10].

CD95 (also known as Apo1 or Fas) is a membrane receptor belonging to the tumor necrosis factor receptor superfamily, which leads to apoptosis when combined with its ligand the transmembrane protein FasL (or CD95L) [11]. CD95 is believed to be expressed in injured spinal cord, and treatments reducing expression of the marker after trauma may represent promising therapies for SCI [11,12].

The hyperosmolar agent mannitol has been widely employed in the treatment of brain swelling after traumatic brain injury. In this regard, improvements in microcirculation and brain perfusion, as well as inhibition of free radicals have been confirmed after mannitol administration. Nevertheless, few studies have reported use of mannitol for SCI $[13,14]$. Against this background, the aim of the present study was to analyse the ability of hyperosmolar solution administration to reduce apoptosis in rats submitted to SCI by clipping at the Th10 spinal cord level.

\section{Material and Methods}

\section{Animals}

A total of 30 adult female Wistar rats (205-235g in body weight) were used. All experimental procedures were performed in accordance with the guidelines proposed by MASCIS (Multicenter Animal Spinal Cord Injury Study), and with national and institutional guidelines for animal experimentation, after approval by the Research Ethics Committee of the Evangelical College of Medicine of Curitiba.

\section{Spinal cord injury model}

The clipping compression method was used to induce spinal cord injury, whose efficacy for producing moderate to severe partial spinal lesion at the Th10 level has been proven by several authors $[15,16]$. To this end, animals were anaesthetized with an intraperitoneal injection of KETAMINE (Merck, Rio de Janeiro, Brazil, $0.75 \mathrm{mg} /$ $\mathrm{kg}$ body weight) and CHLORPROMAZINE (Merck, $0.2 \mathrm{mg} / 100$ $\mathrm{g}$ body weight). Prophylactically, rats were given $0.25 \mathrm{mg} / 10 \mathrm{~g}$ of CEFALOTIN (ABL, Cosmópolis, Brazil) intraperitoneally. Before clipping, the thoracic spinal cord was exposed by laminectomy at T8-T12 using a microsurgical technique. Temporary aneurysm clips (Aesculap, Tutlingen, Germany) were previously calibrated to $35 \mathrm{~g}$ and applied to the cord without opening the dura, and closed for 1 minute. Postoperatively, $0.1 \mathrm{~mL}$ of BUPRENORPHINE (Andrômaco, Lisbon, Portugal) in $5 \mathrm{~mL}$ saline solution was administered for proper analgesia.

\section{Administration of mannitol solution}

MANNITOL solution at 20\% (Merck) and $2 \mathrm{~g} / \mathrm{kg}$ of body weight, was administered as a bolus infusion intraperitoneally in one group of animals immediately after surgery, and repeated every 3 hours for 72 hours.

\section{Animals were divided into 3 experimental groups as follows:}

Group 1: laminectomy, spinal cord clipping, and mannitol solution;

Group 2: laminectomy and spinal cord clipping

Group 3: laminectomy only

\section{Post-operative care}

After surgery, animals were transferred to their cages and kept at room temperature $\left(27^{\circ} \mathrm{C}\right)$. Bladder emptying was performed manually three times daily and $2.5 \mathrm{mg} / 5 \mathrm{~mL}$ gentamycin solution was given intraperitoneally once a day. Animals were kept alive for 3 days post-

*Corresponding author: Guilherme Lepski, Hoppe Seyler Strasse 3, 72076 Tuebingen, Germany, Tel: +49 707129 86679; Fax: +49 707129 5245; E-mail: lepski@gmail.com

Received May 05, 2011; Accepted October 21, 2011; Published October 24 2011

Citation: Dobrowolski S, Rassier GI, Lepski G (2011) Hyperosmolar Solution Reduces Apoptosis in the Acute Spinal Cord Injury Model in Rats. J Cytol Histol 2:124. doi:10.4172/2157-7099.1000124

Copyright: () 2011 Dobrowolski S, et al. This is an open-access article distributed under the terms of the Creative Commons Attribution License, which permits unrestricted use, distribution, and reproduction in any medium, provided the original author and source are credited. 
operatively, considered sufficient time for primary and secondary inflammatory changes to take place [11].

\section{Behavioural analysis}

Animals were evaluated by two independent and blinded observers on the BBB motor scale at 24 and 48 hours after spinal cord lesion [17]. The evaluation was performed at night, in synchrony with the circadian activity cycle of rodents.

\section{Animal perfusion and histology}

At 72 hours after surgery, the animals were killed by inhalation of sulfuric ether, and the carotid-jugular compartment perfused with $500 \mathrm{~mL}$ cold PBS (Invitrogen/Gibco, Sao Paulo, Brazil), followed by $500 \mathrm{~mL}$ fresh, cold 4\% PARAFORMALDEHYDE (Sigma Aldrich, Sao Paulo, Brazil). The vertebral column from C5 to L5 was resected, and the exact site of the surgical lesion was visualized under light microscope, and then cut $2 \mathrm{~cm}$ above and $2 \mathrm{~cm}$ below this level. The section of interest was washed in buffered 10\% PARAFORMALDEHYDE and embedded in paraffin. The tissue was cut into $5 \mu \mathrm{m}$-thick slices in the axial plane $0.5 \mathrm{~cm}$ cranially, and $0.5 \mathrm{~cm}$ caudally, from the lesion site, with $0.5 \mathrm{~mm}$ space between slices. Slices were anatomically arranged and each spinal cord section was subdivided into 9 parts for further analysis, as follows: 1. white matter: anterior right, anterior left, posterolateral right, posterolateral left and posterior, and 2. gray matter: anterior left and right, posterior left and right.

First, standard HEMATOXYLIN/EOSIN staining was performed. Briefly, sections were deparaffinized in XYLENE in 3 washes of 10 minutes each. Subsequently, sections were re-hydrated in absolute alcohol for 10 minutes, $95 \%$ alcohol for 5 minutes, $85 \%$ for 5 minutes and finally, $70 \%$ alcohol for 5 minutes. Sections were then washed under tap water for 5 minutes. This was followed by incubation in HARRIS HEMATOXYLIN solution for 20 minutes, a further wash in running tap water for 20 minutes and incubation with eosin for 1 minute. Finally, sections were dehydrated by brief washing in $85 \%$ alcohol, then for 5 minutes in $95 \%$ alcohol, and 3 washes of 10 minutes each in absolute alcohol, cleared in 3 washes of XYLENE for 5 minutes. Specimens were then mounted in xylene-based mounting medium.

Second, tissues were stained with luxol fast blue (LFB). For staining, sections were deparaffinized and hydrated in 95\% alcohol, left in $0.1 \%$ LUXOL FAST BLUE solution (diluted in 95\% alcohol) at $56^{\circ} \mathrm{C}$ overnight, then rinsed in $95 \%$ ethyl alcohol and distilled water. Slides were then differentiated in $0.05 \%$ lithium carbonate solution (in distilled water) for 30 seconds, and in 70\% ethyl alcohol for an additional 30 seconds. Finally, slices were rinsed again in distilled water, counterstained in $0.1 \%$ cresyl violet (diluted in distilled water) for 40 seconds, rinsed again in distilled water, incubated in 95\% ethyl alcohol for 5 minutes and in 100\% alcohol twice for 5 minutes. After double washes in xylene (5 minutes), preparations were mounted in resinous medium. All reagents were purchased from Sigma Aldrich (Sao Paulo, Brazil)

Specimens were analyzed for 4 criteria established for acute spinal cord injury by Black et al. [18], as follows:

1- White matter degeneration, characterized by edema, formation of cysts, demyelination and infiltration of macrophages, cystic necrosis and cytoarchitectonic disorganization.

\section{2- Hemorrhage in white or gray matter;}

3- Neuronal loss, sometimes with vacuolization and inflammatory infiltration in gray matter;

4- Signs of hypoxic injury: nuclear retraction and pyknosis, as well as intense eosinophilic staining of the pericardium.

Based on the above-described criteria, histological alterations related to the intensity of necrosis were classified semi-quantitatively into the following four categories after scanning of all slices: $<1 \%$ of total scanned area: score $0 ; 1-24 \%$ of total area: score $1 ; 25-49 \%$ : score $2 ; 50-74 \%$ : 3 ; and finally $>75 \%$ : score 4 . This quantification was performed by two independent and experienced pathologists on two different sections in a blinded manner and without knowledge of the experimental group.

\section{Immunohistochemistry}

Expression of CD95 was revealed by streptavidin-biotinperoxidase. To this end, paraffin-embedded $3 \mu \mathrm{m}$-thick slices were arranged on glass coverslips, washed in xylene for 15 minutes, then in PBS ( $\mathrm{pH}$ 7.3-7.4), and citrate-buffer $(\mathrm{pH} \mathrm{6.0)}$ ) for 30 minutes at $95^{\circ} \mathrm{C}$. Thereafter, slices were positioned for 15 minutes in blocks with endogen activated peroxidase with $3 \% \mathrm{H}_{2} \mathrm{O}_{2}$ and methanol. Slices were then washed again in PBS.

The primary anti-CD95 monoclonal rat antibody was diluted $1: 100$ and incubated overnight at $37^{\circ} \mathrm{C}$ for 60 minutes over a shaker, and the slices were again rinsed in PBS and incubated for 60 minutes with biotinylated secondary antibody (polyclonal rabbit anti-rat, 1:400, Dako, Denmark) at $37^{\circ} \mathrm{C}$. After successive rinses in PBS, another 60-minute incubation with streptavidin-peroxidase complex (streptavidin/horseradish peroxidase, 1:600, Dako, Denmark) and aminoethylcarbazole Sigma Aldrich, Sao Paulo, Brazil) at $37^{\circ} \mathrm{C}$ was performed.

\section{Quantification and statistical analysis}

Under an optical light microscope with a magnification $\mathrm{x} 100$, the percentage of CD95-positive cells in relation to the total nuclear count was determined for 3 slices from each animal at the level of the lesion. Using morphometry and image analysis with a macro application, the total number of cells in the gray and white matter (on the basis of the number of nuclei in each histological section), and the number of cells clearly showing positive immunostaining for CD95 were recorded and averaged. Quantifications were performed by two independent researchers trained in morphometric determinations, who had no knowledge of the experimental group from which each sample was obtained. For CD95 quantifications, a score of 0 was attributed when less than $1 \%$ of the total cells were positively stained, a score of 1 if $1-24 \%$ of the total cells were positive, a score of 2 for $25-49 \%$ positivity, a score of 3 for $50-74 \%$ staining, and finally a score of 4 if more than $75 \%$ of the cells stained for CD95. Data are expressed as mean \pm standard error means (s.e.m.). The standard deviation between observers is additionally shown in the data Tables $(1,2,3)$. Means were compared among groups with the Mann-Whitney U non-parametric statistical test. All statistical analyses were performed with the SPSS Software package version 13.0 (SPSS Inc., Chicago, IL, USA). Statistical significance is indicated in the Figures and Tables as follows: ${ }^{*}$ for $\mathrm{p}<0.05,{ }^{* *}$ for $\mathrm{p}<0.01$, and ${ }^{* * *}$ for $\mathrm{p}<0.001$.

\section{Results}

In the animal model employed, based on temporary clipping of the thoracic spinal cord, marked necrosis was evident in the white and 
Citation: Dobrowolski S, Rassier GI, Lepski G (2011) Hyperosmolar Solution Reduces Apoptosis in the Acute Spinal Cord Injury Model in Rats. J Cytol Histol 2:124. doi:10.4172/2157-7099.1000124

Page 3 of 6

\begin{tabular}{|c|c|c|c|c|c|c|}
\hline \multicolumn{7}{|l|}{ BBB scores } \\
\hline \multirow[t]{2}{*}{ rat } & \multicolumn{2}{|c|}{ control } & \multicolumn{2}{|c|}{ sham } & \multicolumn{2}{|c|}{ mannitol } \\
\hline & observer 1 & observer 2 & observer 1 & observer 2 & observer 1 & observer 2 \\
\hline 1 & 21 & 21 & 0 & 0 & 0 & 0 \\
\hline 2 & 21 & 21 & 2 & 2 & 0 & 0 \\
\hline 3 & 21 & 21 & 1 & 1 & 1 & 1 \\
\hline 4 & 21 & 21 & 0 & 0 & 2 & 2 \\
\hline 5 & 21 & 21 & 0 & 0 & 1 & 1 \\
\hline 6 & 21 & 21 & 1 & 1 & 0 & 0 \\
\hline 7 & 21 & 21 & 2 & 2 & 2 & 2 \\
\hline 8 & 21 & 21 & 0 & 0 & 1 & 1 \\
\hline 9 & 21 & 21 & 1 & 1 & 1 & 1 \\
\hline 10 & 21 & 21 & 1 & 1 & 0 & 0 \\
\hline mean 1 & 21 & 21 & 0.8 & 0.8 & 0.8 & 0.8 \\
\hline SD bet. obs. & \multicolumn{2}{|c|}{0} & \multicolumn{2}{|c|}{0} & \multicolumn{2}{|c|}{0} \\
\hline tot. mean & \multicolumn{2}{|c|}{21} & \multicolumn{2}{|c|}{0.8} & \multicolumn{2}{|c|}{0.8} \\
\hline s.e.m. & \multicolumn{2}{|c|}{0.000} & \multicolumn{2}{|c|}{0.249} & \multicolumn{2}{|c|}{0.249} \\
\hline$p$-value & & & & & \multicolumn{2}{|c|}{1.0} \\
\hline
\end{tabular}

Table 1: BBB scores in the experimental groups.

\begin{tabular}{|c|c|c|c|c|c|c|c|c|c|c|c|c|}
\hline \multicolumn{13}{|c|}{ necrosis estimation } \\
\hline \multirow[b]{3}{*}{ rat } & \multicolumn{4}{|c|}{ control } & \multicolumn{4}{|c|}{ sham } & \multicolumn{4}{|c|}{ mannitol } \\
\hline & \multicolumn{2}{|c|}{ white matter } & \multicolumn{2}{|c|}{ gray matter } & \multicolumn{2}{|c|}{ white matter } & \multicolumn{2}{|c|}{ gray matter } & \multicolumn{2}{|c|}{ white matter } & \multicolumn{2}{|c|}{ gray matter } \\
\hline & observer 1 & observer 2 & observer 1 & observer 2 & observer 1 & observer 2 & observer 1 & observer 2 & observer 1 & observer 2 & observer 1 & observer 2 \\
\hline 1 & 0 & 0 & 0 & 0 & 5.2 & 4.3 & 5.3 & 4.2 & 2.9 & 4.1 & 3.6 & 3.6 \\
\hline 2 & 0 & 0 & 0 & 0 & 3.7 & 2.8 & 3.4 & 3.7 & 3.6 & 4.5 & 2 & 2.9 \\
\hline 3 & 0 & 0 & 0 & 0 & 4.3 & 4.2 & 3.8 & 2.9 & 3.9 & 3.9 & 2.8 & 2.6 \\
\hline 4 & 0 & 0 & 0 & 0 & 3.9 & 3.4 & 4.4 & 4.2 & 4 & 4.6 & 4 & 4.2 \\
\hline 5 & 0 & 0 & 0 & 0 & 4.5 & 4.8 & 2.9 & 3.6 & 3.4 & 2.9 & 4.4 & 4.1 \\
\hline 6 & 0 & 0 & 0 & 0 & 2.8 & 3.7 & 4.4 & 4.9 & 4.7 & 5.5 & 2.9 & 3.2 \\
\hline 7 & 0 & 0 & 0 & 0 & 3.5 & 4.1 & 5.2 & 4.1 & 4.2 & 4.3 & 3.1 & 2.7 \\
\hline 8 & 0 & 0 & 0 & 0 & 4 & 3.3 & 3.8 & 3.8 & 3.8 & 4 & 2.4 & 3.9 \\
\hline 9 & 0 & 0 & 0 & 0 & 4 & 3.5 & 3 & 3.6 & 3.8 & 4.4 & 3.3 & 3.3 \\
\hline 10 & 0 & 0 & 0 & 0 & 4.1 & 3.9 & 4.8 & 4 & 3.7 & 3.8 & 3.5 & 3.5 \\
\hline mean 1 & 0 & 0 & 0 & 0 & 4 & 3.8 & 4.1 & 3.9 & 3.8 & 4.2 & 3.2 & 3.4 \\
\hline SD betw. obs. & \multicolumn{2}{|c|}{0} & \multicolumn{2}{|c|}{0} & \multicolumn{2}{|c|}{0.1} & \multicolumn{2}{|c|}{0.1} & \multicolumn{2}{|c|}{0.2} & \multicolumn{2}{|c|}{0.1} \\
\hline tot. mean & \multicolumn{2}{|c|}{0.0} & \multicolumn{2}{|c|}{0.0} & \multicolumn{2}{|c|}{4.0} & \multicolumn{2}{|c|}{4.1} & \multicolumn{2}{|c|}{3.8} & \multicolumn{2}{|c|}{3.2} \\
\hline s.e.m. & \multicolumn{2}{|c|}{0.00} & \multicolumn{2}{|c|}{0.00} & \multicolumn{2}{|c|}{0.20} & \multicolumn{2}{|c|}{0.27} & \multicolumn{2}{|c|}{0.15} & \multicolumn{2}{|c|}{0.23} \\
\hline p-value & & & & & & & & & & 209 & & 028 \\
\hline
\end{tabular}

Table 2: Estimation of necrosis based on hematoxylin-eosin stainings. 
Citation: Dobrowolski S, Rassier GI, Lepski G (2011) Hyperosmolar Solution Reduces Apoptosis in the Acute Spinal Cord Injury Model in Rats. J Cytol Histol 2:124. doi:10.4172/2157-7099.1000124

Page 4 of 6

\begin{tabular}{|c|c|c|c|c|c|c|c|c|c|c|c|c|}
\hline \multicolumn{13}{|c|}{ CD95 quantifications } \\
\hline \multirow[b]{3}{*}{ rat } & \multicolumn{4}{|c|}{ control } & \multicolumn{4}{|c|}{ sham } & \multicolumn{4}{|c|}{ mannitol } \\
\hline & \multicolumn{2}{|c|}{ white matter } & \multicolumn{2}{|c|}{ gray matter } & \multicolumn{2}{|c|}{ white matter } & \multicolumn{2}{|c|}{ gray matter } & \multicolumn{2}{|c|}{ white matter } & \multicolumn{2}{|c|}{ gray matter } \\
\hline & observer 1 & observer 2 & observer 1 & observer 2 & observer 1 & observer 2 & observer 1 & observer 2 & observer 1 & observer 2 & observer 1 & observer 2 \\
\hline 1 & 0 & 0 & 0 & 0 & 0.8 & 1.1 & 3.1 & 2.9 & 1.3 & 0.9 & 1.6 & 1 \\
\hline 2 & 0 & 0 & 0 & 0 & 2.1 & 1.9 & 2.3 & 2.1 & 0.8 & 0.6 & 2.1 & 1.3 \\
\hline 3 & 0 & 0 & 0 & 0 & 1.7 & 1.7 & 1.8 & 1.7 & 0.6 & 0.4 & 1.8 & 0.9 \\
\hline 4 & 0 & 0 & 0 & 0 & 1.5 & 0.7 & 3.4 & 2.7 & 1.2 & 1 & 1.3 & 1.3 \\
\hline 5 & 0 & 0 & 0 & 0 & 1.2 & 1.1 & 2.8 & 2.4 & 1.5 & 0.8 & 1.4 & 1.1 \\
\hline 6 & 0 & 0 & 0 & 0 & 1.9 & 1.5 & 2.4 & 1.9 & 0.7 & 1.2 & 2 & 1.7 \\
\hline 7 & 0 & 0 & 0 & 0 & 1.7 & 1.3 & 2.2 & 2.4 & 0.5 & 0.7 & 1.5 & 0.8 \\
\hline 8 & 0 & 0 & 0 & 0 & 2.2 & 1.5 & 3 & 3.1 & 1.1 & 0.8 & 1.9 & 1.5 \\
\hline 9 & 0 & 0 & 0 & 0 & 1.3 & 0.9 & 2.1 & 2.2 & 1.4 & 0.9 & 0.9 & 1.6 \\
\hline 10 & 0 & 0 & 0 & 0 & 2.6 & 1.3 & 2.9 & 2.6 & 0.9 & 0.7 & 2.5 & 1.8 \\
\hline mean 1 & 0 & 0 & 0 & 0 & 1.7 & 1.3 & 2.6 & 2.4 & 1 & 0.8 & 1.7 & 1.3 \\
\hline SD bet. obs. & \multicolumn{2}{|c|}{0} & \multicolumn{2}{|c|}{0} & \multicolumn{2}{|c|}{0.2} & \multicolumn{2}{|c|}{0.1} & \multicolumn{2}{|c|}{0.1} & \multicolumn{2}{|c|}{0.2} \\
\hline tot. mean & \multicolumn{2}{|c|}{0.0} & \multicolumn{2}{|c|}{0.0} & \multicolumn{2}{|c|}{1.7} & \multicolumn{2}{|c|}{2.6} & \multicolumn{2}{|c|}{1.0} & \multicolumn{2}{|c|}{1.7} \\
\hline s.e.m. & \multicolumn{2}{|c|}{0.00} & \multicolumn{2}{|c|}{0.00} & \multicolumn{2}{|c|}{0.17} & \multicolumn{2}{|c|}{0.16} & \multicolumn{2}{|c|}{0.11} & \multicolumn{2}{|c|}{0.14} \\
\hline$p$-value & & & & & & & & & 6.60 & E-05 & 2.00 & 0E-06 \\
\hline
\end{tabular}

Table 3: Quantification of CD95-positivity.

gray matter at the level of the injury. The necrosis was characterized by neuronal loss, vacuolization, pyknotic nuclei and micro-hemorrhages, cytoarchitectonic disorganization, and axonal swelling (Figure 1). Immediately after trauma, the animals were unable to move their hind limbs and only slight movements of one or two joints could be observed
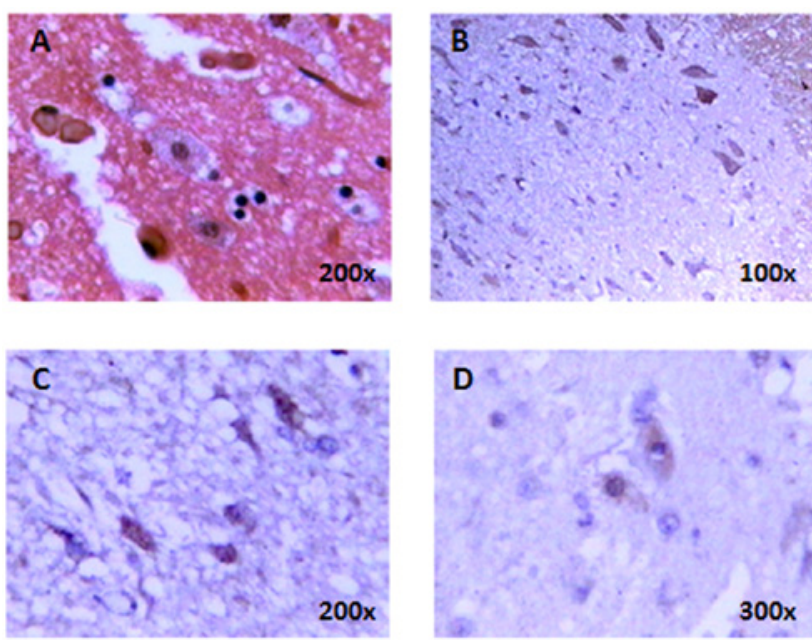

Figure 1: Spinal cord injury through clipping induces necrosis and CD95 expression. In A, white matter of the thoracic spinal cord of injured rat 3 days after lesion. Coagulation and/or liquefaction necrosis evident in the lesioned thoracic segment, with cytoarchitectonic disorganization, axonal swelling, neuronal loss, vacuolization, hemorrhage, and pyknotic nuclei. In B and C, CD95 staining in white matter (control group after clipping). In D, CD95 staining in white matter of animal from the mannitol group.
(BBB 0 or 1), thus characterizing complete or severe partial lesion.

\section{Motor tests}

Regarding restoration of motor function according to the $\mathrm{BBB}$ scale, no differences were observed between treated and lesioned-nontreated groups in the acute phase three days after lesion (Figure 2A). Specifically, scores of $0.8 \pm 0.17$ were recorded in the mannitol group, $0.8 \pm 0.17$ in the sham group, and $21 \pm 0$ in the control group.

\section{Necrosis quantification}

In the experimental groups with spinal cord injury, necrosis was analyzed semi-quantitatively (Figure $2 \mathrm{~B}$ ). Lower necrosis scores were found in gray matter in the group of animals treated with mannitol ( $3.3 \pm 0.14$ versus $4.0 \pm 0.16, \mathrm{p}<0.01$ in the sham group, $\mathrm{n}=10$ ). For white matter, the corresponding values were not statistically different, perhaps because the neuronal cell number was considerably lower ( $4.0 \pm 0.13$ versus $3.9 \pm 0.13, \mathrm{p}=0.616, \mathrm{n}=10)$. Histological evaluation revealed normal tissue in the control group.

\section{CD95 expression}

The expression of CD95 was further analysed, representing a reliable marker of apoptosis (Figure 2C). CD95 was highly expressed in the lesion site, but not above or below it. In the white matter, CD95-expression was considerably higher in the sham group: $1.5 \pm$ 0.11 versus $0.9 \pm 0.07$ in the group treated with mannitol $(\mathrm{p}<0.001)$. Correspondingly, CD95 expression in gray matter was only $1.5 \pm 0.10$ in the treated group, compared with $2.5 \pm 0.11$ in the sham group $(\mathrm{p}<0.001)$. CD95-expression was not detected in the control group. 

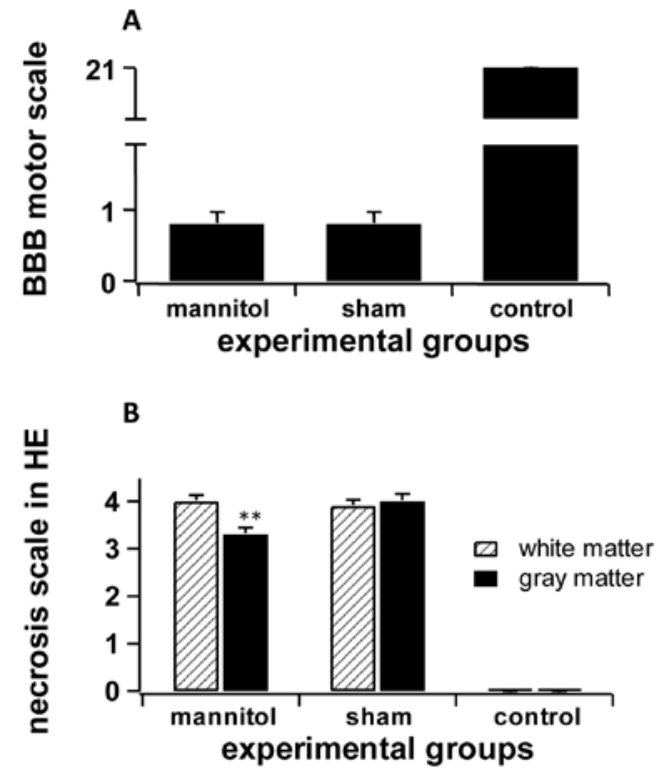

c

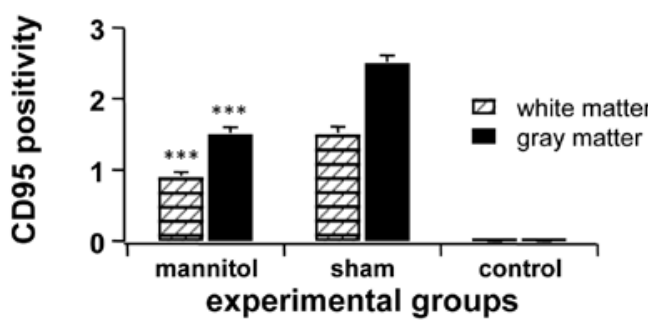

Figure 2: Mannitol solution reduced necrosis and CD95 positivity after $\mathrm{SCl}$. In A, regarding motor recovery, no improvement was observed within the initial 3 days after acute spinal cord injury; In B, semi-quantitative analysis of hematoxylin-eosin stained samples, according to the criteria in the methods, revealed significant reduction in degree of necrosis in gray matter in the experimental group that received mannitol. In white matter, no significan change was observed, perhaps due to the low number of cells. In C, semiquantitative analysis of CD95-positivity revealed significantly fewer positive cells in white and gray matter in the mannitol group.

\section{Discussion}

The acute phase after spinal cord injury is of utmost relevance since numerous pathological processes take place during this period, in which cell death is the hallmark feature. It is now assumed that cell death after trauma is caused by both necrosis and apoptosis, i.e., two distinct pathophysiological processes. Necrosis is caused by passive cell death and is independent of cellular control. It is characterized by a cytotoxic edema, which results in membrane rupture and secondary inflammatory reaction due to liberation of cytoplasmatic content. By contrast, apoptosis represents a mechanism of physiological regulation under genetic influence. In spinal cord injury, apoptosis can affect neurons as well as glial cells. Although many of the triggering mechanisms of apoptosis are largely unknown, there is a large body of evidence supporting an important role of glutamate, calcium, free radicals, nitric oxide, and numerous protein factors such as Fasligands[19-22].

It is believed that trauma-related apoptosis is not only responsible for the secondary degeneration at the lesion level, but may also be responsible for the chronic demyelination that occurs at sites distal to the CNS in late follow up [23].

The transmembrane glycoprotein CD95 has $48 \mathrm{KD}$ and is a member of the superfamily of NGF-receptors. CD95 is considered one of the most important mediators of apoptosis, inducing cell death once bound to the Fas/APO1 ligand (a cytotoxic cytokine homologous to TNF). Apoptosis can be induced by a variety of different stimuli, besides the interaction between CD95 (Fas/APO1) and its ligand, resulting in a cascade of reactions. In this regard, the involvement of CD95 in the apoptosis of certain activated T-cells (Paneth cells) is well known, but its implication in the apoptosis of other cell types remains obscure, largely because expression of CD95 in other tissues has yet to be elucidated [11,24].

According to recent evidence, CD95 expression reaches a peak between 8 and 24 hours after CNS-lesion, in both white and gray matter [11]. Based on this information, our study focused on the acute phase of the SCI model, and demonstrated a positive effect of hyperosmolar solution on the reduction of CD95 expression compared to controls, 72 hours after trauma.

\section{The mechanism of action of mannitol can be explained by}

i) osmotic dehydration of brain tissue with reduction of interstitial water content; ii) increase in cerebral blood flow (CBF) due to transient hypervolemia; iii) reduction of blood viscosity due to hemodilution; and finally iv) a rheologic effect related to alterations in erythrocyte morphology. Combined, these effects contribute to better oxygensupply to the brain tissue, which in turn allows a reduction in cerebral blood volume $(\mathrm{CBV})$, and consequently a lowering of intracranial pressure (ICP) $[13,25]$. Nevertheless, mannitol has been implicated in the inhibition of free radicals. Its role as a scavenger of free radicals, especially from hydroxyl-radicals, has been reported by several authors [13,25-27]. According to these authors, mannitol is able to induce a transient but significant increase in cardiac output and a marked reduction in hematocrit $[25,27]$. The effect of mannitol in the treatment of SCI has been analyzed only by a few research groups. Some evidence supports the idea that mannitol prevents secondary lesions when applied during the acute phase after trauma. Nevertheless, distinct animal models and evaluation methods hamper result interpretation and no consensus can yet be reached.[13,28] Our results reinforce this initial concept by demonstrating a significant reduction in CD95 expression in neuronal and glial cells in the acute SCI model in rats. Although the mechanisms of action of mannitol are still not fully understood, its use in acute SCI may represent an effective approach to rescue viable neural cells in damaged spinal cord. Inhibition of secondary apoptosis constitutes a central issue in the management of SCI and may represent a promising new hope for victims of this devastating condition. Further studies are warranted to demonstrate the degree of functional recovery achieved using this strategy, as well as the potential benefit in human patients. Our results represent a small step toward achieving this goal.

\section{Acknowledgements}

This work was supported with grants from FAPESP (Sao Paulo Research Foundation, Brazil) and CNPq (National Council for Scientific and Technological Development, Brazil). The authors would like to thank Dr. Analía Arévalo for assistance with the final editing of the manuscript. 
Citation: Dobrowolski S, Rassier GI, Lepski G (2011) Hyperosmolar Solution Reduces Apoptosis in the Acute Spinal Cord Injury Model in Rats. J Cytol Histol 2:124. doi:10.4172/2157-7099.1000124

\section{References}

1. Behrmann DL, Bresnahan JC, Beattie MS (1994) Modeling of acute spinal cord injury in the rat: neuroprotection and enhanced recovery with methylprednisolone, U-74006F and YM-14673. Exp Neurol 126: 61-75.

2. Faden Al, Salzman S (1992) Pharmacological strategies in CNS trauma. Trends Pharmacol.Sci. 13: 29-35.

3. Ho A, Chi JH (2010) Early surgery recommended for acute central cord injury. Neurosurgery 67: N21-N22.

4. Balentine JD (1978) Pathology of experimental spinal cord trauma. I. The necrotic lesion as a function of vascular injury. Lab Invest 39: 236-253.

5. Balentine JD (1978) Pathology of experimental spinal cord trauma. II Ultrastructure of axons and myelin. Lab Invest 39: 254-266.

6. Goodkin R and Campbell JB (1969) Sequential pathologic changes in spina cord injury: a preliminary report. Surg.Forum 20: 430-432.

7. Holtz A, Nystrom B, Gerdin B (1989) Spinal cord blood flow measured by 14C-iodoantipyrine autoradiography during and after graded spinal cord compression in rats. Surg Neurol 31: 350-360

8. Noble LJ, Wrathall JR (1989) Distribution and time course of protein extravasation in the rat spinal cord after contusive injury. Brain Res 482: 57-66.

9. Tator $\mathrm{CH}$ and Fehlings MG (1991) Review of the secondary injury theory of acute spinal cord trauma with emphasis on vascular mechanisms. J Neurosurg 75: $15-26$

10. Faden Al, Ellison JA, Noble LJ (1990) Effects of competitive and noncompetitive NMDA receptor antagonists in spinal cord injury. Eur $\mathrm{J}$ Pharmacol 175: 165-174.

11. Zurita M, Vaquero J, Zurita I (2001) Presence and significance of CD-95 (Fas APO1) expression after spinal cord injury. J Neurosurg 94: 257-264.

12. Austin JW, Fehlings MG (2008) Molecular mechanisms of Fas-mediated cell death in oligodendrocytes. J Neurotrauma 25: 411-426.

13. Baysefer A, Erdogan E, Kahraman S, Izci Y, Korkmaz C, et al. (2003) Effect of mannitol in experimental spinal cord injury: an ultrastructural and electrophysiological study. Neurol India 51: 350-354.

14. Magnaes B (1977) Effect of mannitol in a patient with spinal cord injury: a case report. Acta Neurochir (Wien) 39: 59-61.

15. Fehlings M , Nashmi R (1995) Assessment of axonal dysfunction in an in vitro model of acute compressive injury to adult rat spinal cord axons. Brain Res 677: 291-299.
16. Seki T, Fehlings MG (2008) Mechanistic insights into posttraumatic syringomyelia based on a novel in vivo animal model. Laboratory investigation. J Neurosurg Spine 8: 365-375.

17. Basso DM, Beattie MS, Bresnahan JC (1995) A sensitive and reliable locomotor rating scale for open field testing in rats. J Neurotrauma 12: 1-21.

18. Black P, Markowitz RS, Cooper V, Mechanic A, Kushner H, et al. (1986) Models of spinal cord injury: Part 1. Static load technique. Neurosurgery 19: 752-762.

19. Crowe MJ, Bresnahan JC, Shuman SL, Masters JN, Beattie MS (1997) Apoptosis and delayed degeneration after spinal cord injury in rats and monkeys. Nat Med 3: 73-76.

20. Emery E, Aldana P, Bunge MB, Puckett W, Srinivasan A, et al. (1998) Apoptosis after traumatic human spinal cord injury. J Neurosurg 89: 911-920.

21. Li Y, Sharov VG, Jiang N, Zaloga C, Sabbah HN, et al. (1995) Ultrastructural and light microscopic evidence of apoptosis after middle cerebral artery occlusion in the rat. Am J Pathol 146: 1045-1051.

22. Rieger J, Stander M, Loschmann PA, Heneka M, Dichgans J, et al. (1998) Synthesis and biological effects of $\mathrm{NO}$ in malignant glioma cells: modulation by cytokines including CD95L and TGF-beta, dexamethasone, and p53 gene transfer. Oncogene 17: 2323-2332.

23. Panter SS, Yum SW, Faden Al (1990) Alteration in extracellular amino acids after traumatic spinal cord injury. Ann Neurol 27: 96-99.

24. Demjen D, Klussmann S, Kleber S, Zuliani C, Stieltjes B, et al. (2004) Neutralization of CD95 ligand promotes regeneration and functional recovery after spinal cord injury. Nat Med 10: 389-395.

25. Reed JE, Allen WE 3rd, Dohrmann GJ (1979) Effect of mannitol on the traumatized spinal cord. Microangiography, blood flow patterns, and electrophysiology. Spine (Phila Pa 1976) 4: 391-397.

26. Dyste GN, Hitchon PW, Girton RA, Chapman M (1989) Effect of hetastarch mannitol, and phenylephrine on spinal cord blood flow following experimental spinal injury. Neurosurgery 24: 228-235

27. Kaptanoglu E, Palaoglu S, Surucu HS, Hayran M, Beskonakli E (2002) Ultrastructural scoring of graded acute spinal cord injury in the rat. J Neurosurg 97: 49-56

28. Isu T (1990) [Spinal cord evoked potential in experimental spinal cord injurythe changes in spinal cord evoked potential following impact injury, and effect of mannitol administration on acute experimental spinal cord injury]. Hokkaido Igaku Zasshi 65: 142-151. 